\title{
Conocimientos financieros en estudiantes universitarios
}

Financial knowledge in university students

Mg. Juan Jose

Yaringaño Limache

Docente de la Universidad

Marcelino Champagnat jyaringano@umch.edu.pe

Recepción 2/06/2018

Revisado $10 / 06 / 2018$

Aceptación $22 / 08 / 2018$

\section{Resumen}

$\mathrm{n}$ el mundo de hoy se observa incremento de $\checkmark$ diversos productos financieros y crediticios; estos plantean la necesidad de las personas por adquirir conocimientos en conceptos financieros básicos, lo cual les permitirá tomar decisiones informadas con respecto al ahorro, la inversión o el endeudamiento (Klapper,Lusardiy Oudheusden,2015). El presente estudio es de tipo descriptivo comparativo y tiene como objetivo identificar los conocimientos financieros en los estudiantes universitarios y verificar si se presentan diferencias significativas al compararlos según las variables sexo, edad, carrera profesional, estado civil, tener hijos, tener trabajo y tarjeta de débito o crédito. La muestra estuvo conformada por 176 estudiantes de ambos sexos y pertenecientes a las carreras de Administración, Contabilidad, Educación y Psicología de la Universidad Marcelino Champagnat. Los resultados indicaron que un $47.8 \%$ obtuvo un puntaje de 3 a más puntos lo cual indica que se tienen los conocimientos básicos a nivel financiero, siendo los estudiantes de áreas relacionadas con aspectos administrativos los que presentan un promedio mayor, de modo significativo, en relación a las otras carreras.

Palabras clave: conocimientos financieros, estudiantes universitarios. 


\begin{abstract}
Tn today's world there is an increase in diverse financial and credit products, which raise the need for people to acquire knowledge in basic financial concepts which will allow them to make informed decisions regarding savings, investment or indebtedness (Klapper, Lusardi \& Oudheusden, 2015). The present study of comparative descriptive type, aims to identify financial knowledge in university students and compare if there are significant differences when comparing them according to the variables sex, age, career, marital status, having children, having a job and having a card debit or credit. The sample consisted of 176 students of both sexes and belonging to the careers of Administration, Accounting, Education and Psychology of the Marcelino Champagnat University. The results indicated that $47.8 \%$ obtained a score of 3 to more points which indicates that they have the basic knowledge at a financial level, being the students from areas related to administrative aspects those that present a higher average in a significant way in relation to the others careers.
\end{abstract}

Key words: financial knowledge, university students. 


\section{Introducción}

En el contexto socio económico actual, donde se observa un incremento de los productos financieros y crediticios cada vez más sofisticados, se plantea la necesidad de promover cambios positivos en el comportamiento económico y una mejora en los niveles de educación financiera de los individuos y las familias. Existe evidencia de que los bajos niveles de educación financiera conlleva a efectos negativos (OECD, 2005). Las personas con un conocimiento adecuado de conceptos financieros básicos tienen la capacidad de tomar decisiones informadas con respecto al ahorro, la inversión, el endeudamiento y más (Klapper, Lusardi, \& van Oudheusden, 2015).

El desconocimiento de conceptos financieros se puede circunscribir en un proceso más amplio, como es el caso de la inclusión financiera, la cual es definida como el acceso y uso de servicios financieros de calidad por parte de todos los segmentos de la población (Graham, 2013), dentro de un proceso de promoción de un acceso asequible, oportuno y adecuado a una amplia gama de productos y servicios financieros regulados y la ampliación de su uso por todos los segmentos de la sociedad; esto incluye actividades de sensibilización y de educación financiera, con el fin de promover el bienestar económico y la inclusión económica y social (García, Grifoni, López y Mejía, 2013).

De este modo, el acceso a los servicios financieros de calidad, se ha convertido en la Estrategia Nacional de Inclusión Financiera (Comisión Multisectorial de Inclusión Financiera, 2015), el cual se convierte en un instrumento de la política de Estado, con el fin de ejecutar acciones coordinadas que contribuyan al desarrollo económico descentralizado e inclusivo, con la participación del sector público y privado, dentro del contexto de estabilidad financiera, siendo fundamental lograr que la persona desarrolle una cultura financiera o alfabetismo financiero (Financial Literacy), en especial los jóvenes, ya que son ellos los usuarios de modo continuo del sector financiero. Un aspecto esencial es conocer el nivel conocimientos o de cultura financiera; para ello se han desarrollado diversas encuestas para medir esta variable (Lusardi, 2015).

Debemos considerar que nuestro modelo económico promueve productos financieros considerando que los individuos manejan la información adecuada para tomar decisiones correctas. Sin embargo, Lusardi y Mitchell (2011) enfatizan que el analfabetismo financiero se presenta con mayor medida en 
la población joven o en la tercera edad, dado que son financieramente poco sofisticados; esto hace necesario alfabetizar financieramente a los jóvenes desde los últimos años de la educación secundaria, y en especial al inicio de la educación universitaria, ya que es la etapa en la que inician sus contactos con el mundo financiero (Vi-llagómez, 2016).

Una cuestión asociada a la educación financiera es quien es responsable de ella; según Olin (2014) los medios más utilizados por los ciudadanos son el autoaprendizaje y obtener información de las personas más cercanas, pero estos medios no son necesariamente los más prácticos, confiables y efectivos. Por lo tanto, señala que el gobierno, a nivel local y nacional, así como las instituciones privadas, tanto financieras como no financieras, deben llevar a cabo a través de los medios de comunicación una mayor difusión para la concientización y fomento de una educación financiera efectiva y de calidad.

Para conocer los niveles de cultura financiera básica, se ha desarrollado un cuestionario con cuatro preguntas denominado Global Financial Literacy Survey; en el cuestionario original se consideró que contestar correctamente tres preguntas de cuatro, indicaba tener conocimientos financieros básicos. En la versión española la última pregunta se separó en dos preguntas, pero manteniendo el criterio de acertar tres para aprobar (Villavicencio, 2015). Este cuestionario originalmente fue elaborado para determinar el nivel de alfabetización financiera de la población mundial. La encuesta fue utilizada con más de 150.000 adultos de 140 países, las preguntas trataban sobre la tasa de interés simple y compuesto, la inflación y la diversificación de riesgos; los resultados en España, indicaron que solo el $49 \%$ de los encuestados aprobaron.

El presente trabajo se planteó dos objetivos: el primero, conocer el nivel de conocimientos financieros en los estudiantes universitarios y comparar si se presentan diferencias significativas al comparar las variables sexo, edad, carrera profesional, estado civil, tener hijos, tener trabajo y tener tarjeta de débito o crédito. El segundo objetivo se refiere a hacer participar a los estudiantes del proceso de recolección y análisis de datos, con el fin de desarrollar las competencias asociadas a la investigación. 


\section{Método}

Tipo y diseño de investigación. La investigación es de tipo cuantitativa y descriptivo comparativa. Se utilizó un diseño no experimental transversal, describiéndose los puntajes obtenidos en cultura financiera según las variables socio demográficas: sexo, edad, carrera profesional, estado civil, tener hijos, tener trabajo y tener tarjeta de débito o crédito.

Sujetos. La muestra de estudio estuvo conformada por 176 sujetos de ambos sexos y pertenecientes a las diferentes carreras de la Universidad Marcelino Champagnat. En la Tabla 1, se describe la conformación de la muestra según carrera y sexo.

Tabla 1

Descripción de la muestra

\begin{tabular}{|c|c|c|c|}
\hline \multicolumn{4}{|c|}{ Sexo } \\
\hline Carrera & Masculino & Femenino & Total \\
\hline Administración & 20 & 27 & 47 \\
\hline Contabilidad & 11 & 15 & 26 \\
\hline Educación & 21 & 30 & 51 \\
\hline Psicología & 19 & 33 & 52 \\
\hline Total & 71 & 105 & 176 \\
\hline
\end{tabular}

Muestreo. Se realizó un muestreo no probabilístico intencional, pues se solicitó a los estudiantes universitarios su consentimiento para poder completar el cuestionario.

Instrumento de recolección de datos. El instrumento para evaluar los conocimientos financieros (Romainville, 2015) de los alumnos se encuentra en el apéndice. Está conformado por dos partes. En la primera se realiza una encuesta sociodemográfica y en la segunda se presenta un cuestionario de 5 preguntas en relación a conocimientos financieros. Las preguntas se presentan a continuación. 


\section{Preguntas:}

1. Suponga que cuenta con algunos ahorros y decide montar un negocio. ¿Destinarías toda tu inversión a un negocio o a varios?

Las alternativas: A. Un negocio, B. Varios negocios y C. No lo sé

2. Imagina que en los próximos diez años los precios de los productos se duplican. Si tu sueldo también se multiplica por dos. ¿Puedes comprar más cosas? ¿Menos? ¿Las mismas?

Las alternativas: A. Menos, B. Las mismas, C. Más y D. No lo sé

3. Si te prestan 100 soles, ¿Con cuál de estas ofertas tendrás que devolver menos?

Las alternativas: A. 100 soles más el 3\%, B. 105 soles y C. No lo sé

4. Imagina que depositas tu dinero en tu banco durante dos años en una cuenta que no puedes mover y tu banco te ofrece a cambio unos intereses del $15 \%$ cada año. ¿Te darán más dinero el segundo año que el primero?

Las alternativas: A. No sé, B. No, me darán el mismo, C. Sí

5. Imagina que depositas 100 soles en tu cuenta bancaria y el banco te añade el $10 \%$ cada año. Después de cinco años, si no has sacado nada de dinero tendrás...

Las alternativas: A. Más de 150 soles, B. Menos de 150 soles, C. 150 soles

La primera interrogante del cuestionario se refiere a diversificación de riesgos, la segunda se relaciona con la inflación, la tercera se debe utilizar la aritmética y las dos últimas al interés compuesto.

Análisis estadístico. El análisis estadístico se realizó utilizando medidas de tendencia central (Media), estadísticos de dispersión (Desviación estándar y varianza), así como la comparación de las medias de los grupos observados (U de Mann Whitney y Kruskall Wallis). 


\section{Resultados}

A continuación, se presentan los resultados obtenidos en la encuesta de acuerdo con diferentes variables sociodemográficas

Se observa en la Tabla 2 que los alumnos de administración son aquellos obtienen un mayor promedio (Media $=2.81$ ) en conocimientos financieros, además se aprecia que son el grupo con puntaje menos disperso ( $\mathrm{CV}=42.4 \%)$ en relación a los otros. A su vez son los alumnos de sexo masculino los que presentan mayor promedio (Media $=2.59$ ), pero son las alumnas las que presentan una menor dispersión $(\mathrm{CV}=52.3 \%)$. Por otro, los estudiantes que trabajan tienen una media mayor (Media $=2.50$ ) pero presentan a su vez una mayor dispersión de los puntajes obtenidos $(\mathrm{CV}=54.8 \%)$. También se observó que los estudiantes que tienen tarjeta de crédito o débito son los que presentan una media mayor (Media=2.56) pero a su vez tienen una mayor dispersión de los puntajes obtenidos $(\mathrm{CV}=55.1 \%)$. Finalmente son los estudiantes que mantiene una relación de convivencia los que obtuvieron un mayor puntaje promedio (Media=2.75) y son los que presentan una menor dispersión de los puntajes obtenidos $(\mathrm{CV}=38.4 \%)$.

\section{Tabla 2}

Promedio en el cuestionario de conocimiento financieros según variables sociodemográficas

\begin{tabular}{lcccc}
\hline & & Media & Desv. Est. & CV \\
\hline \multirow{3}{*}{ Carrera profesional } & Administración & 2,81 & 1,190 & $42.4 \%$ \\
& Contabilidad & 2,30 & 1,350 & $58.7 \%$ \\
& Educación & 2,21 & 1,270 & $57.5 \%$ \\
& Psicología & 2,37 & 1,347 & $56.8 \%$ \\
\hline \multirow{2}{*}{ Sexo } & Masculino & 2,59 & 1,489 & $57.5 \%$ \\
& Femenino & 2,32 & 1,213 & $52.3 \%$ \\
\hline \multirow{2}{*}{ Condición laboral } & Trabaja & 2.50 & 1.369 & $54.8 \%$ \\
& No Trabaja & 2.35 & 1.255 & $53.4 \%$ \\
\hline \multirow{2}{*}{$\begin{array}{l}\text { Tenencia de tarjeta de } \\
\text { crédito o debito }\end{array}$} & Tiene tarjeta & 2,56 & 1,411 & $55.1 \%$ \\
\hline
\end{tabular}




\begin{tabular}{lcccc}
\hline & Soltero & 2,43 & 1,356 & $55.8 \%$ \\
Estado civil & Casado & 2,27 & 1,104 & $48.6 \%$ \\
& Conviviente & 2,75 & 1,055 & $38.4 \%$ \\
\hline
\end{tabular}

Prueba de normalidad. La Tabla 3, presenta los niveles críticos (Sig. = Significación). De la prueba Kolmogorov-Smirnov se observa que los datos de la muestra en la variable, tiene asociados niveles críticos menores que 0,05 , lo que debe llevarnos a concluir que los datos obtenidos, no proceden de una población con distribución normal.

\section{Tabla 3}

Pruebas de normalidad

\begin{tabular}{lccc}
\hline & \multicolumn{3}{c}{ Kolmogorov - Smirnov } \\
\cline { 2 - 4 } Total & Estadístico & $\mathrm{gl}$ & Sig. \\
\cline { 2 - 4 } &, 196 & 90 &, 000 \\
\hline
\end{tabular}

De acuerdo con los resultados de la Tabla 3 se decide el uso de estadísticos no paramétricos para comprobar si las diferencias de medias observadas según las variables de comparación son significativas.

La tabla 4 presenta el estadístico U de Mann-Whitney, la tipificación Z y el nivel crítico (Sig. asintót.). Se observan niveles críticos bilaterales mayores a 0.05 en todos los casos. Por tanto, no podemos rechazar la hipótesis de igualdad de promedios y concluir que estos grupos definidos por las variables sexo, tener hijos, tener trabajo y tener tarjeta de crédito o débito proceden de poblaciones con similar promedio.

Tabla 4

Prueba U de Mann-Whitney

\begin{tabular}{lcccc}
\hline $\begin{array}{c}\text { Variables de } \\
\text { comparación }\end{array}$ & $\begin{array}{c}\text { U de Mann- } \\
\text { Whitney }\end{array}$ & W de Wilco-xon & Z & $\begin{array}{c}\text { Sig. asintótica } \\
\text { (bilateral) }\end{array}$ \\
\hline Sexo & 804.500 & 2182.500 & -1.547 & .122 \\
Hijos & 567.000 & 757.000 & -1.013 & .311 \\
Trabajo & 896.500 & 1676.500 & -.670 & .503 \\
Tarjeta & 758.000 & 1083.000 & -.507 & .612 \\
\hline
\end{tabular}


La Tabla 5 contiene el estadístico de Kruskal-Wallis (Chi-cuadrado), sus grados de libertad ( $g l)$ y su nivel crítico (Sig. asintót.). Puesto que los niveles críticos son mayores que 0,05 , según el estado civil pero menores a 0,05 en el caso de la carrera profesional. Por lo tanto, podemos rechazar la hipótesis de igualdad de promedios poblacionales y concluir que la muestra comparada según carrera profesional difieren en conocimientos financieros y por contrario si se compara según estado civil no difieren en conocimientos financieros.

\section{Tabla 5}

\section{Prueba de Kruskall-Wallis}

\begin{tabular}{cccc}
\hline & Chi-cuadrado & gl & Sig. asintótica \\
\hline Carrera Profesional & 1.618 & 3 & .045 \\
Estado civil & 1.314 & 2 & .518 \\
\hline
\end{tabular}

Por lo tanto, podemos afirmar que las diferencias observadas son significativas en el caso de los estudiantes de administración y contabilidad en comparación con los grupos de psicología y educación.

Cuando se analiza los resultados según las preguntas y el porcentaje de respuestas obtenidas se observan los siguientes. En la Tabla 6, la pregunta 2 obtiene el mayor promedio (Media $=0,61$ ), lo cual indicaría que se tienen conocimiento en relación a los efectos del aumento del nivel de precios existentes en un mercado durante un período de tiempo sobre la capacidad adquisitiva. Por el contrario, la pregunta 4 presenta el menor promedio (Media $=0,33)$ esto indica déficit en conocimientos en relación a reconocer el beneficio asociada a un capital inicial de acuerdo con un interés que se obtienen al finalizar un periodo determinado de tiempo comparando los beneficios en años diferentes.

Tabla 6

Promedio de respuestas en las preguntas y total

\begin{tabular}{lcccccc}
\hline & Pregunta 1 & Pregunta 2 & Pregunta 3 & Pregunta 4 & Pregunta 5 & Total \\
\hline $\mathrm{N}$ & 178 & 178 & 178 & 178 & 178 & 178 \\
Media & 0,35 & 0,61 & 0,40 & 0,33 & 0,54 & 2,43 \\
Desv. típ. & 0,478 & 0,490 & 0,492 & 0,472 & 0,500 & 1,327 \\
\hline
\end{tabular}


Además, se observa en la Tabla 7 el porcentaje de respuestas obtenidas en los conocimientos financieros de los alumnos, el 52.2\% se encuentra por debajo del puntaje esperado de 3 puntos, lo cual indica que un $47.8 \%$ tiene conocimientos básicos a nivel financiero, siendo los estudiantes de áreas relacionadas con aspectos administrativos los que presentan un promedio cercano a los 3 puntos (Tabla 2).

Tabla 7

Porcentaje de respuestas correctas del total

\begin{tabular}{|c|c|c|c|}
\hline Puntos & F & $\%$ & Porcentaje acumulado \\
\hline 0 & 15 & $8,4 \%$ & $8,4 \%$ \\
\hline 1 & 28 & $15,7 \%$ & $24,2 \%$ \\
\hline 2 & 50 & $28,1 \%$ & $52,2 \%$ \\
\hline 3 & 48 & $27,0 \%$ & $79,2 \%$ \\
\hline 4 & 25 & $14,0 \%$ & $93,3 \%$ \\
\hline 5 & 12 & $6,7 \%$ & $100,0 \%$ \\
\hline
\end{tabular}

Finalmente en la Tabla 8 , se observa que un $63.8 \%$ de los estudiantes de administración ob-tienen 3 puntos sobre el porcentaje mayor al de las otras carreras.

Tabla 8

Porcentaje de respuestas correctas del total según carrera profesional

\begin{tabular}{ccccccccc}
\hline \multirow{2}{*}{ Puntos } & \multicolumn{2}{c}{ Administración } & \multicolumn{2}{c}{ Contabilidad } & \multicolumn{2}{c}{ Educación } & \multicolumn{2}{c}{ Psicología } \\
\cline { 2 - 9 } & F & $\%$ & F & $\%$ & F & $\%$ & F & $\%$ \\
\hline 0 & 5 & $10.6 \%$ & 2 & $7.4 \%$ & 5 & $9.6 \%$ & 3 & $5.8 \%$ \\
1 & 4 & $8.5 \%$ & 6 & $22.2 \%$ & 10 & $19.2 \%$ & 8 & $15.4 \%$ \\
2 & 8 & $17.0 \%$ & 8 & $29.6 \%$ & 16 & $30.8 \%$ & 18 & $34.6 \%$ \\
3 & 13 & $27.7 \%$ & 6 & $22.2 \%$ & 13 & $25.0 \%$ & 16 & $30.8 \%$ \\
4 & 12 & $25.5 \%$ & 3 & $11.1 \%$ & 6 & $11.5 \%$ & 4 & $7.7 \%$ \\
5 & 5 & $10.6 \%$ & 2 & $7.4 \%$ & 2 & $3.8 \%$ & 3 & $5.8 \%$ \\
\hline
\end{tabular}




\section{Discusión}

El presente estudio se planteó conocer de manera descriptiva si el nivel de conocimientos en aspectos financieros está condicionado o influido por el tipo de carrera de estudio, la condición laboral, el sexo, la tenencia de tarjeta de crédito o débito y el estado civil. Estos resultados evidencian que el contexto académico en los cuales se desarrollan los alumnos favorece la adquisición de un mayor conocimiento de aspectos financieros, y que aún se deben mejorar, pero que esto también, como contrapartida, define que es necesaria una inclusión más exitosa en el uso de los servicios financieros cada vez más sofisticados o complejos a los estudiantes de profesiones diversas.

Se observa en los resultados, que los alumnos presentan mayor nivel de conocimientos en relación con los efectos que puede tener en el aumento de los precios existentes en un mer-cado durante un período de tiempo, sobre la capacidad adquisitiva de las personas. Pero, por el contrario, presentan menor nivel de conocimientos en relación a reconocer el beneficio asociado a tener capital inicial depositado con un interés, durante un periodo determinado de tiempo y comparar a su vez esos beneficios en años distintos.

Estos resultados descriptivos ponen en evidencia que se requiere el desarrollo de capacidades en los alumnos universitarios para la implementación y ejecución de estrategias personales y para la selección de alternativas y la toma de decisiones valorativas que permiten el mejor uso de servicios financieros.

Como señala Olin (2014), desarrollar estas competencias requiere comprender conceptos y adquirir conocimientos, desarrollar habilidades, actitudes y capacidades para una adecuada toma de decisiones, referente a la administración de los recursos económicos y el uso efectivo de los productos y servicios financieros, dado que esto repercute en el bienestar de las personas.

El desarrollo de programas de educación financiera según (Aguilar \& Ortiz, 2013), debe considerar que la necesidad de educarse financieramente es un área fundamental en los jóvenes, por lo que es necesario enseñarles a cuidar, planificar y valorar el dinero, y en el caso de los adolescentes se convertiría en una visión para el futuro, cuando estos formen parte del mundo laboral y profesional. Por lo tanto, los programas de educación y cultura financiera que se requieran implementar desde el sector público o privado se deben enfocar 
en aspectos como: la planeación para la vida, el ahorro de los ingresos o la planificación de la inversión.

\section{Referencias}

Aguilar \& Ortiz, (2013). Diseño de un programa de educación y cultura financiera para los estudiantes de modalidad presencial de la titulación en administración en banca y finanzas. (Tesis de titulación). Universidad de Loja, Ecuador.

Comisión Multisectorial de Inclusión Financiera (2015). Estrategia Nacional de Inclusión Financiera. Lima: Ministerio de Economía y Finanzas. Recuperado de https://www.mef.gob.pe/contenidos/archivos-descarga/ ENIF.pdf

García, N., Grifoni, A., Lopez, J. y Mejía, M. (2013). La educación financiera en América Latina y el Caribe. Situación actual y perspectivas. Caracas: Banco de desarrollo de América Latina.

Klapper, L, Lusardi, A. \& van Oudheusden, P. (2015). Financial Literacy Around the World: Insights from the standard \& poor's ratings services global financial literacy survey. Standard and Poor's. Recuperado de http://www.FinLit.MHFI.com

Lusardi, A. (2015). Financial literacy: Do people know the ABCs of finance? Public Understanding of Science, 24(3), 260-271.

Lusardi, A. \& O. Mitchell O. (2011). Financial Literacy and Planning: Implications for Retirement Wellbeing", en O. S. Mitchell y A. Lusardi (eds.), Financial Literacy: Implications for Retirement Security and the Financial Marketplace, Oxford University Press, Oxford.

Olin, B. (2014). La educación financiera como base para la toma de decisiones personales de inversión. (Tesis de Maestría). Universidad Autónoma de Querétaro, México. 
Romainville, M. (20 noviembre 2015). Test: Descubre aquí si eres un analfabeto financiero. Diario El Comercio.

Varcoe, K. et al., (2005). Using a financial education curriculum for teens. Association for Financial Counseling and Planning Education. 16(1): 63-71.

Villagómez, F. (2016). Alfabetismo financiero en jóvenes preparatorianos en la Zona Metropolitana del Valle de México. El trimestre económico, 83(3), 331, 677-706.

Villavicencio, Y. (20 noviembre 2015). ¿Aprobarías el examen de cultura financiera? Son solo cinco preguntas. El País. Recuperado de https:// elpais.com/economia/2015/11/19/actualidad/1447957909_428797. html 


\section{Apéndice}

Test de conocimiento financiero

\section{Primera parte: Encuesta}

Carrera $\quad: \square$ Administración $\square$ Contabilidad $\square$ Educación $\square$

Psicología

Edad

Sexo : $\square$ Masculino $\square$ Femenino

Estado civil : $\square$ Soltero $\square$ Casado $\square$ Conviviente

Tiene hijos $\quad: \square \mathrm{Si} \quad \square$ No

Trabaja actualmente: $\square$ Si $\square$ No

Cuenta con tarjeta de crédito o débito $\quad: \square \mathrm{Si} \quad \square$ No

Considera que hace buen uso de su tarjeta : $\square \mathrm{Si} \quad \square$ No

Según su opinión ¿Qué es Educación financiera?

Según su opinión ¿Quién estaría encargado de enseñar Educación financiera?

Según su opinión ¿A qué edad y dónde se debería enseñar la Educación financiera?

\section{Segunda parte: Cuestionario}

1. Suponga que cuenta con algunos ahorros y decide montar un negocio. ¿Destinarías toda tu inversión a un negocio o a varios?
A. Un negocio
B. Varios negocios
C. No lo sé 
2. Imagina que en los próximos diez años los precios de los productos se duplican. Si tu sueldo también se multiplica por dos. ¿Puedes comprar más cosas? ¿Menos? ¿Las mismas?
A. Menos
B. Las mismas
C. Más
D. No lo sé

3. Si te prestan 100 soles, ¿Con cuál de estas ofertas tendrás que devolver menos?
A. 100 soles más el 3\%
B. 105 soles
C. No lo sé

4. Imagina que depositas tu dinero en tu banco durante dos años en una cuenta que no puedes mover y tu banco te ofrece a cambio unos intereses del $15 \%$ cada año. ¿Te darán más dinero el segundo año que el primero?
A. No sé
B. No, me darán el mismo
C. Sí

5. Imagina que depositas 100 soles en tu cuenta bancaria y el banco te añade el $10 \%$ cada año. Después de cinco años, si no has sacado nada de dinero tendrás...
A. Más de 150 soles
B. Menos de 150 soles
C. 150 soles 